\title{
Editorial: Computational Defect Properties
}

\author{
Shijun Zhao ${ }^{1 *}$, Yongfeng Zhang $^{2}$, Luca Messina ${ }^{3}$ and Christian Brandl ${ }^{4}$ \\ ${ }^{1}$ Department of Mechanical Engineering, City University of Hong Kong, Kowloon, Hong Kong, ${ }^{2}$ Department of Engineering \\ Physics, University of Wisconsin, Madison, WI, United States, ${ }^{3} C E A, D E S$, IRESNE, DEC-Service d'Études et de Simulation du \\ Comportement des Combustibles, Saint-Paul-Lez-Durance, France, ${ }^{4}$ Department of Mechanical Engineering, University of \\ Melbourne, Parkville, VIC, Australia
}

Keywords: defect properties, numerical simulations, computational materials sciences, nuclear materials, radiation damage

Editorial on the Research Topic

\section{Computational Defect Properties}

A century of studies has demonstrated that many properties of materials are controlled predominantly by defects or faults in the crystal structures. Indeed, defects are major carriers that mediate atomic and mass transport and influence the structural stability and performance of materials. Line defects such as dislocations are central to the mechanical properties of materials and crystal growth. Dopant defects are decisive ingredients in the modern silicon-based semiconductor industry. Solid-state lasers, battery technologies, and solid oxide fuel cells all rely on the characteristics of impurities and/or defects. Therefore, the knowledge of defect properties is of vital importance for understanding materials performance and further for rational materials design. Thanks to the development of advanced experimental characterization techniques and computer simulation methodologies, the field of defects has witnessed massive development in recent years. In this hand-in-hand progress, simulations have been an indispensable tool to gain insight into the process invisible to experiment equipment. This is exactly the purpose of this Research Topic, where we focus on the latest development in computational defect properties based on state-of-the-art theoretical approaches.

The topics touched upon in this collection involve basic defect energetics, defect-induced strain, defect diffusion, the surface and size effects on defect properties, and radiation-induced segregation. It spans over a wide range of materials, including structural materials such as $\mathrm{W}$ and Ni-based alloys, nuclear fuels, and novel high entropy alloys. Besides, various computational techniques are introduced, ranging from electronic-level density functional theory calculations, atomic-level molecular dynamics to the continuum-level rate theory models. Notably, the combination of these methods with machine learning techniques is successfully demonstrated.

First, the work of Beeler et al. represented a thorough evaluation of the defects in $\alpha$ phase of Uranium, the fundamental source of nuclear fuel, based on ab initio molecular dynamics (AIMD) at finite temperatures. The $\alpha$ phase with face-centered orthorhombic structure is the favorable form of U below $935 \mathrm{~K}$. The calculated temperature-dependent thermophysical properties, including lattice parameters, thermal expansion, and heat capacity, exhibit a transition, with rapid changes below $400 \mathrm{~K}$, followed by a moderate change. Temperature-dependent point defect formation energies and induced lattice strains were also determined to shed light on the experimentally observed irradiationinduced growth. It was found that interstitials cause significantly higher strain than vacancies, and that strain is strongly dependent on lattice directions, where the $a$ direction expands and the $b$ contracts. The direction of point defect-induced lattice strain is contrary to the irradiation growth behavior of $\alpha-\mathrm{U}$. These results lay the foundation for mesoscale simulations for $\alpha-\mathrm{U}$ in which defect populations, defect diffusion, and defect strain behaviors come into play together. 
Another work by Manzoor et al. reported the application of machine learning in predicting the point defect energies in multiprincipal element alloys (MPEAs), including high-entropy alloys (HEAs). In MPEAs, defect energies are highly dependent on the local atomic environments due to the chemical disorder. This work showed that machine learning built upon a database of constituent binary alloys can be used to predict vacancy migration and formation energies in face-centered cubic ternary, quaternary, and quinary alloys in the $\mathrm{Ni}-\mathrm{Fe}-\mathrm{Cr}-\mathrm{Co}-\mathrm{Cu}$ system. Such machine learning methodology may enable rapid assessment of defect energies in MPEAs, which is extremely helpful to understand and tune their irradiation performance.

The contribution of Sun et al. focused on the effect of strain on the diffusion of both near-surface self-interstitials (SIAs) and adatoms in tungsten (W), the most promising candidate material for the plasma-facing components and divertor in fusion reactors. Based on molecular dynamics, the authors investigated the average time required for an SIA in the center of a tungsten slab to a (110) surface at different strains and temperatures. The results suggested that strain significantly modifies the propensity for SIAs to reach the surface, and the fraction of them that forms dislocation loops in the bulk crystal. Adatom diffusion is also strongly affected by strain, resulting in strain-dependent surface roughness evolution.

$\mathrm{Hu}$ et al. presented a microstructure-dependent rate theory model for radiation-induced segregation (RIS) in binary alloys, in which the effects of inhomogeneous thermodynamic and kinetics properties of defects were explicitly considered. Using four independent variables to describe the system, including atom $A$, interstitial A, interstitial B, and vacancy on $[A, B]$ sublattice, the spatial characteristics of defect generation and diffusion were accounted for. The developed model reveals the effects of defect chemical potentials and mobilities on RIS in polycrystalline $\mathrm{AB}$ model alloys.

The work of Ozturk et al. focused on the surface and grain size effects on the transient and steady-state behaviors of point defects in irradiated pure $\mathrm{Ni}$ and binary $\mathrm{Ni}-\mathrm{Cr}$ based on a spatially resolved rate-theory modeling approach. In this model, the influences of dose rate, production bias, defects recombination, reactions with volumetric sinks, and diffusion to surface sinks are examined. It is found that surface and size always exert effects on defect behavior in the presence of production bias. The author showed that this is related to a surface-induced and size-regulated instability triggered above a critical size between 100 and $500 \mathrm{~nm}$, which favors the separation of vacancies and interstitials. Such patterns induce anomalies in the dependence of defect concentrations and sink strength on grain size, leading to more severe radiation damage. In alloys, the magnitude and range of elemental enrichment/depletion at the boundaries increase with size.

Finally, Vizoso and Deo reported molecular dynamics simulations on the dependence of vacancy formation energies in $\gamma \mathrm{U}-\mathrm{Zr}$ alloys at $0 \mathrm{~K}$ on the first nearest neighbor configurations with varying $\mathrm{Zr}$ concentrations. Comparisons between random populated systems with special quasi-random structures show that there is only a slight change in statistically averaged vacancy formation energies, indicating the dominant role of local atomic environments on vacancy properties. Therefore, adequate sampling of the local configurations is the key to get a full understanding of defect properties in alloys.

We hope this Research Topic will attract readers working in the field of defects and stimulate novel research ideas.

\section{AUTHOR CONTRIBUTIONS}

SZ wrote the first draft of the manuscript. All authors contributed to manuscript revision, read, and approved the submitted version.

Conflict of Interest: The authors declare that the research was conducted in the absence of any commercial or financial relationships that could be construed as a potential conflict of interest.

Publisher's Note: All claims expressed in this article are solely those of the authors and do not necessarily represent those of their affiliated organizations, or those of the publisher, the editors and the reviewers. Any product that may be evaluated in this article, or claim that may be made by its manufacturer, is not guaranteed or endorsed by the publisher.

Copyright (C) 2021 Zhao, Zhang, Messina and Brandl. This is an open-access article distributed under the terms of the Creative Commons Attribution License (CC BY). The use, distribution or reproduction in other forums is permitted, provided the original author(s) and the copyright owner(s) are credited and that the original publication in this journal is cited, in accordance with accepted academic practice. No use, distribution or reproduction is permitted which does not comply with these terms. 